\title{
Comportamento e preferência alimentar em Podocnemis expansa (Schweigger), $P$. unifilis (Troschel) e $P$. sextuberculata (Cornalia) em cativeiro (Testudines, Pelomedusidae)
}

\author{
Adriana Malvasio ${ }^{1}$, Ana Maria de Souza ${ }^{2}$, \\ Flavio de Barros Molina ${ }^{3} \&$ Francisco de Arruda Sampaio ${ }^{4}$
}

\author{
${ }^{1}$ Campus Universitário de Araguaína, Fundação Universidade do Tocantins. Rua Humberto de Campos 508, 77807-060 \\ Araguaina, Tocantins, Brasil. E-mail: drimalvasio@uol.com.br \\ 2 Departamento de Zoologia, Instituto de Biociências, Universidade de São Paulo. Rua do Matão, Travessa 14, 101, \\ 05508-900 São Paulo, Brasil. E-mail: amsouza@ib.usp.br \\ 3 Seção de Répteis, Fundação Parque Zoológico de São Paulo. Avenida Miguel Stefano 4241, 04301-905 Água Funda, São \\ Paulo, Brasil. E-mail: fbmolina@originet.com.br \\ ${ }_{4}^{4}$ Instituto de Pesquisas Ambientais. Rua David Pimentel 193, 05657-010 São Paulo, Brasil. E-mail: fransampaio@uol.com.br
}

\begin{abstract}
Feeding behavior and food preference of Podocnemis expansa (Schweigger), $P$. unifilis (Troschel) and $P$. sextuberculata (Cornalia) in captivity (Testudines, Pelomedusidae). The feeding behavior and the food preference was studied in P. expansa (Schweigger, 1812) $P$. unifilis (Troschel, 1848) and P. sextuberculata $($ Cornalia, 1849). The method used for feeding behavior and food preference was the sampling of all occurrences. The Students' $t$ test was applied on the food items, to compare differences during the development of each species. The main conclusions are: feeding behavior for food, except alive animals, is divided in foraging, approach, olfactory recognition, capture, laceration and ingestion; the persecution behavior was observed for prey activity; cleptoparasitism occurs in the three species and neustophagia mechanism was detected in $P$. unifilis; $P$. sextuberculata shows to be almost only carnivorous and $P$. expansa and $P$. unifilis show to be omnivorous; $P$. expansa can be considered more herbivorous in captivity than $P$. unifilis during the age between one and five years old and more than five years old; $P$. expansa is more sensitive than $P$. unifilis concerning the alteration of the food place, mainly the meat, decreasing its consumption, if it is put on a dry place.
\end{abstract}

KEY WORDS. Captivity, food behavior, food preference, Podocnemis.

Poucas espécies de Testudines têm seu comportamento alimentar descrito em detalhes (Molina et al. 1998). CARPENTER \& Ferguson (1977) comentam que, como a maioria das espécies de Testudines é aquática, há uma dificuldade de observação do comportamento na natureza, muitas vezes faltando detalhes nas informações. Segundo os autores, o comportamento em répteis é considerado potencialmente estereotipado, o que leva a crer que, em condições adequadas de cativeiro, o padrão comportamental pouco será alterado. Isso é um fator bastante positivo, pois as observações em cativeiro podem ser realizadas de forma detalhada.

Legler (1993) comenta que a maioria das espécies de Testudines é onívora oportunista e apresenta comportamento típico de onívoros aquáticos, rondando perto do leito do rio, investigando a vegetação e as frutas caídas das árvores, além dos botes oportunistas em pequenos invertebrados. Na família Pelomedusidae existem espécies carnívoras e onívoras (Goin \& Goin 1962). Segundo Pritchard \& Trebbau (1984), Podocnemis expansa (Schweigger, 1812), além de uma dieta herbívora, o seu conteúdo estomacal pode apresentar pequena quantidade de alimentos de origem animal, como por exemplo, esponjas. Também P. unifilis (Troschel, 1848), além da dieta herbívora, consome ainda insetos e moluscos.

A dieta pode variar em função do sexo e da idade do indivíduo. Teran et al., (1995) comentam que, após analisar conteúdos estomacais de $P$. unifilis na natureza, encontraram 
diferenças na dieta entre machos e fêmeas. Sementes e frutas eram mais consumidas por fêmeas e talos e brotos pelos machos. Clark \& GibBons (1969) ressaltam uma mudança no hábito alimentar de Trachemys scripta (Schoepff, 1792) na natureza, em função da idade dos exemplares. Os autores apontam que os adultos são herbívoros e carnívoros oportunistas, enquanto os jovens, no primeiro ano de crescimento, têm dieta predominantemente carnívora. HART (1983) também cita alteração alimentar para T. scripta, ressaltando que a mudança na dieta envolve o aumento no consumo de plantas pelos adultos. Moreira \& Loureiro (1992), trabalhando com P. expansa em cativeiro, salientam a alteração do hábito alimentar em indivíduos mais jovens, predominantemente carnívoros, em relação aos mais velhos, predominantemente herbívoros.

Estudos sobre a variação da dieta em função da espécie e da idade contribuem para a criação em cativeiro, bastante incentivada atualmente pelo Centro Nacional dos Quelônios da Amazônia, CENAQUA/IBAMA, que objetiva, principalmente, evitar a retirada de exemplares da natureza para a sua comercialização. Mediante as informações acima, este trabalho apresenta como objetivos acompanhar em $P$. expansa, $P$. unifilis e $P$. sextuberculata (Cornalia, 1849) o comportamento e a preferência alimentar por dieta de origem animal ou vegetal em cativeiro, em função da idade dos indivíduos.

\section{MATERIAL E MÉTODOS}

O acompanhamento do comportamento alimentar foi realizado na Fundação Parque Zoológico de São Paulo. Os animais foram mantidos em caixas d'água de 1000 litros e outros recintos maiores, com área seca e alagada. A temperatura da água variou entre $24^{\circ} \mathrm{C}$ a $30^{\circ} \mathrm{C}$, contendo cada caixa, uma resistência elétrica. Os exemplares de $P$. expansa e $P$. unifilis utilizados eram procedentes da Base Física da Barreira da BemVinda do CENAQUA/IBAMA (atual Centro de Conservação e Manejo de Répteis e Anfíbios - RAN), município de São Miguel do Araguaia - Goiás (Rio Araguaia). Já, os exemplares de $P$. sextuberculata provieram da Base Física do CENAQUA/IBAMA de Santarém, Pará (Rio Amazonas).

A alimentação era fornecida diariamente pela manhã, em torno das 9:30 h. Os alimentos, não necessariamente consumidos na natureza, eram colocados dentro da água e consistiam dos seguintes alimentos: couve, almeirão, repolho, tomate, mamão, laranja, banana, maçã, abóbora, cenoura, folhas de Elodea sp. sardinha, tilápia e carne bovina moída. Eventualmente, utilizavam-se presas vivas como minhocas, grilos ou larvas de Tenebrionidae (Coleóptera).

O experimento sobre preferência por alimentos de origem animal e vegetal foi realizado na Faculdade de Medicina Veterinária do Campus Universitário de Araguaína da Fundação Universidade do Tocantins, em um tanque de cimento com 2,23m de comprimento da aresta, cuja área foi dividida em uma pequena parte seca e outra maior, alagada. Caixas d'água de 1000 litros e bacias com diâmetro de $0,5 \mathrm{~m}$ também foram utilizadas, contendo parte seca e outra alagada. O recinto foi deixado à temperatura ambiente, já que as espécies ocorrem na região. Os exemplares utilizados de $P$. expansa e de $P$. unifilis vieram das bases do CENAQUA/IBAMA do Município de São Miguel do Araguaia, em Goiás (Rio Araguaia) e do Parque Nacional do Araguaia, Ilha do Bananal, em Tocantins (Rio Javaés). Os espécimes de $P$. sextuberculata eram procedentes das
Bases Físicas do CENAQUA/IBAMA de Santarém, Pará (Rio Amazonas) e de Rio Branco, Acre (Rio Purús).

Os alimentos foram fornecidos pela manhã, colocados dentro da água, em torno das 10 horas. Com exceção dos exemplares de $P$. sextuberculata, que receberam uma dieta semelhante à fornecida na Fundação Parque Zoológico de São Paulo, aos representantes de $P$. expansa e de $P$. unifilis foi oferecida uma dieta de carne bovina, couve, tomate, cenoura, mamão e maçã.

O estudo do comportamento e da preferência alimentar foi desenvolvido com base na amostragem de todas as ocorrências (cf. Altmann 1974). Os representantes de P. expansa e $P$. unifilis foram divididos em três grupos etários: G1 - 10 exemplares até um ano; G2 - 10 espécimes entre um e cinco anos e G3 - cinco representantes com idade superior a cinco anos. Em $P$. sextuberculata, foram analisados 14 espécimes com até um ano de idade (G1) e, para o grupo (G2), o experimento começou com 10 indivíduos e terminou com dois espécimes, devido a problemas com a manutenção em cativeiro desses exemplares. Foram realizadas 50 horas de observação para cada grupo etário de cada espécie.

Na avaliação da preferência entre alimentos de origem animal e vegetal na dieta os itens alimentares foram cortados em cubos de tamanhos semelhantes e cada cubo foi considerado uma unidade de alimento. Foram contadas as unidades fornecidas e as que restavam ao final de cada período de observação, obtendo-se, com isso, o total de unidades consumidas. $\mathrm{O}$ número de unidades fornecidas para $P$. expansa e $P$. unifilis foi sempre o mesmo: 50 unidades de carne, 10 de couve, 10 de tomate, 10 de cenoura, 10 de mamão e 10 de maçã, para cada período observado. Para obter-se o valor em massa de cada unidade de alimento, foi realizada uma amostragem contendo 100 pedaços de carne e 30 de couve, tomate, cenoura, mamão e maçã, pesados um a um. A massa foi verificada com balança digital de precisão $0,01 \mathrm{~g}$. Após a realização dos experimentos onde contavam-se as unidades de alimento inicial e final, o número consumido de unidades de alimento foi convertido em gramas (Tabs I e II). Em P. sextuberculata, foi mantido o cálculo em unidades (Tabs X e XI).

Foram realizados experimentos com os exemplares de faixa etária entre um a cinco anos de $P$. unifilis e de $P$. expansa, para avaliar se o consumo de alimentos varia em função de colocá-los na água (área alagada), que seria o normal para as espécies aquáticas, ou colocá-los fora da água (área seca). Esta avaliação é importante para as criações comerciais, pois os técnicos do RAN recomendam colocar os alimentos fora da água com o intuito de mantê-la mais limpa, facilitando o manejo.

Nos procedimentos estatísticos, foi aplicado o teste $t$ de Student para comparação dos valores absolutos (em gramas) e relativos (porcentagem) dos alimentos ingeridos nas três faixas etárias (G1, G2, e G3) de P. expansa e P. unifilis. A comparação foi realizada entre as diferentes faixas etárias dentro de uma mesma espécie e na mesma faixa etária entre as duas espécies. Para $P$. sextuberculata, o teste $\mathrm{t}$ de Student não foi aplicado, devido à evidente diferença qualitativa e quantitativa no padrão alimentar desta espécie, em relação às outras. Seguindo SoKAL \& Rohlf (1981), foram considerados significativos (S) os resultados com probabilidade menor que $0,05(\mathrm{p}<0,05)$, muito significativo (SS) quando $\mathrm{p}<0,01$ e altamente significativo (SSS) quando $\mathrm{p}<0,001$. Valores com $\mathrm{p}>0,05$ foram considerados não significativos (NS). 
Tabela I. Massa em gramas dos alimentos fornecidos para representantes de $P$. expansa e de $P$. unifilis até cinco anos de idade. (N) Número de pedaços, (DP) desvio padrão, (Pi) porção individual.

\begin{tabular}{lrccccc}
\hline & Carne & Couve & Tomate & Cenoura & Mamão & Maçã \\
\hline $\mathrm{N}$ & 100 & 30 & 30 & 30 & 30 & 30 \\
Média & 2,49 & 0,14 & 3,82 & 2,05 & 2,85 & 3,06 \\
DP & 0,47 & 0,03 & 1,29 & 0,51 & 0,76 & 0,73 \\
Pi & 124,66 & 1,44 & 38,18 & 20,47 & 28,52 & 30,58 \\
\hline
\end{tabular}

Tabela II. Massa em gramas dos alimentos fornecidos para representantes de $P$. expansa e de $P$. unifilis com mais de cinco anos de idade. (N) Número de pedaços, (desvio $\mathrm{Pd}$ ) desvio padrão, (Pi) porção individual.

\begin{tabular}{lrrrrrr}
\hline & Carne & Couve & Tomate & Cenoura & Mamão & Maçã \\
\hline $\mathrm{N}$ & 100 & 30 & \multicolumn{1}{c}{30} & 30 & 30 & \multicolumn{1}{l}{30} \\
Média & 4,40 & 0,58 & 11,15 & 5,62 & 6,88 & 8,16 \\
DP & 0,95 & 0,16 & 3,14 & 1,64 & 1,23 & 2,34 \\
Pi A232 & 219,89 & 5,85 & 111,45 & 56,18 & 68,76 & 81,60 \\
\hline
\end{tabular}

\section{RESULTADOS}

O comportamento alimentar é semelhante nas três espécies e nas diferentes faixas etárias avaliadas. Quando o alimento é constituído de presas vivas, como por exemplo minhocas, o comportamento engloba as seguintes etapas: forrageio, perseguição, apreensão, dilaceração e ingestão da presa. Essas fases são realizadas na água e podem ocorrer sucessivamente, ou haver interrupção nesta seqüência em função, por exemplo, de desistência da captura ou da dilaceração da presa.

Foi considerado forrageio quando o animal permanece nadando ou caminhando no recinto, aproximando as narinas do solo. A perseguição ocorre quando a presa está movimentando-se na água e o quelônio a localiza. A apreensão caracteriza-se pelo abocanhamento do alimento. Isso pode ocorrer no chão da caixa d'água ou do tanque, ou na superfície da água. Algumas vezes foi observada a apreensão da presa inteira., por sucção. A dilaceração consiste na utilização dos membros dianteiros de forma alternada, usando o membro que estiver do mesmo lado para o qual a cabeça estiver voltada. A ação das garras e da força do membro reparte o alimento em pedaços menores. A ingestão do alimento se dá com o quelônio abrindo e fechando a boca, projetando a cabeça para frente e para trás, respectivamente, caracterizando, assim, uma deglutição gradual.

Quando os alimentos não têm mobilidade (frutas, verduras, pedaços de carne), as fases do comportamento alimentar são as seguintes: forrageio, aproximação, reconhecimento olfativo, apreensão, dilaceração e ingestão. Após localizar o alimento os exemplares realizam a aproximação, submersos, andando no fundo do tanque ou nadando. Ả medida que o animal se aproxima do alimento, pode ocorrer a aceitação ou rejeição do mesmo, provavelmente através da utilização do olfato, pois o animal aproxima as narinas do alimento.

Observou-se, freqüentemente, cleptoparasitismo (um espécime tentando tirar o alimento do outro) em todos os grupos das espécies estudadas. Este comportamento ocorreu mesmo quando havia alimento disponível no recinto. As disputas ocorriam independentemente do tamanho dos indivíduos. Era comum o afastamento de um exemplar com alimento quando outro indivíduo se aproximava dele.

Eventualmente observou-se o comportamento de neustofagia somente nos grupos G2 e G3 de P. unifilis. Este comportamento consiste na ingestão de partículas alimentares suspensas na água (no caso específico estas partículas são aquelas que se desprendem do alimento fornecido), no qual o quelônio mantém o pescoço num ângulo de 45 graus em relação à superfície. Ocorre a abertura da boca por um tempo e, quando esta se fecha, há a ingestão de partículas alimentares

Muitas vezes após a ingestão foi observado outro tipo de comportamento: o exemplar utiliza um dos membros anteriores e o movimenta primeiro sobre a cabeça, indo em direção à boca. Observou-se algumas vezes que através deste movimento o animal retirava restos de carne e gordura da boca.

Deve-se ressaltar que, quando os alimentos foram colocados em área seca para os exemplares de $P$. expansa e de $P$. unifilis pertencentes ao grupo G2, os indivíduos realizavam a etapa de apreensão fora da água, transportando a seguir os alimentos para a água e, portanto, as fases sucessivas de dilaceração e ingestão ocorreram dentro da água.

Com relação à preferência por alimentos de origem animal e vegetal, encontramos a porcentagem dos alimentos consumidos nas diferentes faixas etárias para P.expansa (Tab. III) e para P.unifilis (Tab. IV), assim como os resultados do teste t de Student. A comparação da preferência alimentar entre P.expansa e P.unifilis pertencentes ao mesmo grupo etário, assim como os resultados do teste $t$ de Student, encontram-se nas tabelas V, VI e VII.

Os resultados dos experimentos para avaliar se o consumo de alimentos varia em função de colocá-los na água (área alagada) ou fora da água (área seca), em exemplares do grupo G2 de P.expansa, encontram-se na tabela VIII e os relativos a $P$. unifilis na tabela IX.

O teste t de Student demonstrou que, em $P$. expansa, há um aumento muito significativo $(\mathrm{p}=0,003, \mathrm{SS})$ no consumo de vegetais em função da idade dos indivíduos, quando comparamos o grupo menor de 1 ano (G1) com o grupo entre 1 e 5 anos (G2), cujos alimentos foram colocados na água. Comparando o grupo G1 com o grupo de idade maior de 5 anos (G3), obtém-se um valor altamente significativo ( $p=0,0000001$, SSS) e, na comparação de G2 com G3, também ( $p=0,0002$, SSS) (Tab. III). Isso significa que, com o decorrer da idade, $P$. expansa torna-se mais vegetariana. A média da porcentagem de alimentos de origem vegetal na dieta é $19,1 \%$ no grupo G1, $36,7 \%$ no grupo G2 e $51,6 \%$ em G3.

Em P. unifilis, apesar de ocorrer um aumento na média da porcentagem dos alimentos de origem vegetal, os resultados obtidos não foram estatisticamente significativos, o que indica que esta espécie manteve uma quantidade semelhante de consumo de alimentos vegetais durante seu desenvolvimento (Tab. IV). A média da porcentagem de alimentos de origem vegetal na dieta é $27,4 \%$ no grupo G1, 30,8\% no grupo G2 e $36,8 \%$ em G3. 
A. Malvasio et al.

Tabela III. Porcentagem consumida de vegetais nos diferentes dias de experimento, no decorrer do desenvolvimento de três grupos etários de Podocnemis expansa. (DP) Desvio padrão, (gl) graus de liberdade, (SS) muito significativo, (SSS) altamente significativo.

\begin{tabular}{|c|c|c|c|c|c|c|}
\hline & $<1$ ano & a 5 anos & $<1$ ano & $>5$ anos & 1 a 5 anos & 5 anos \\
\hline & 1,5 & 48,9 & 1,5 & 59,6 & 48,9 & 59,6 \\
\hline & 23,9 & 21,6 & 23,9 & 59,6 & 21,6 & 59,6 \\
\hline & 10,5 & 38,9 & 10,5 & 59,6 & 38,9 & 59,6 \\
\hline & 18,0 & 33,6 & 18,0 & 59,6 & 33,6 & 59,6 \\
\hline & 10,4 & 33,9 & 10,4 & 44,2 & 33,9 & 44,2 \\
\hline & 46,4 & 5,4 & 46,4 & 48,6 & 5,4 & 48,6 \\
\hline & 23,7 & 67,0 & 23,7 & 59,6 & 67,0 & 59,6 \\
\hline & 4,3 & 38,8 & 4,3 & 43,4 & 38,8 & 43,4 \\
\hline & 24,2 & 33,8 & 24,2 & 37,0 & 33,8 & 37,0 \\
\hline & 26,5 & 42,7 & 26,5 & 59,6 & 42,7 & 59,6 \\
\hline & 7,6 & 24,9 & 7,6 & 45,7 & 24,9 & 45,7 \\
\hline & 15,5 & 18,7 & 15,5 & 28,2 & 18,7 & 28,2 \\
\hline & 41,7 & 18,7 & 41,7 & 46,3 & 18,7 & 46,3 \\
\hline & 25,4 & 59,6 & 25,4 & 52,8 & 59,6 & 52,8 \\
\hline & 23,6 & 48,5 & 23,6 & 59,6 & 48,5 & 59,6 \\
\hline & 3,6 & 40,4 & 3,6 & 54,5 & 40,4 & 54,5 \\
\hline & 17,9 & 48,9 & 17,9 & 58,8 & 48,9 & 58,8 \\
\hline Observações & 17 & 17 & 17 & 17 & 17 & 17 \\
\hline Média & 19,1 & 36,7 & 19,1 & 51,6 & 36,7 & 51,6 \\
\hline DP & 12,52 & 15,73 & 12,52 & 9,56 & 15,73 & 9,56 \\
\hline gl & 16 & & 16 & & 16 & \\
\hline Estatística t & $-3,130$ & & $-8,430$ & & $-4,456$ & \\
\hline $\begin{array}{l}\mathrm{P}(\mathrm{T}<=\mathrm{t}) \\
\text { uni-caudal }\end{array}$ & 0,003 SS & & 0,000000 & 11 SSS & 0,0002 SSS & \\
\hline
\end{tabular}

As diferenças dos valores absolutos e percentuais de carne e vegetais consumidos por indivíduos do grupo G1 de $P$. expansa e $P$. unifilis não são significativas, demonstrando que, nesta faixa etária, o consumo desses itens alimentares é semelhante nas duas espécies (Tab. V). A média da porcentagem do consumo de alimentos de origem vegetal é $19,1 \%$ em $P$. expansa e $27,4 \%$ em $P$. unifilis.

A comparação dos resultados do grupo G2 de P. unifilis e de $P$. expansa, cuja alimentação foi colocada na água, revelou um aumento não significativo do consumo de carne, porém, ao mesmo tempo, verificou-se um aumento muito significativo ( $\mathrm{p}=0,005$, SS) do consumo de vegetais por $P$. expansa e um aumento significativo $(\mathrm{p}=0,015, \mathrm{~S})$ na quantidade total de alimentos ingeridos, indicando que $P$. expansa come mais carne
Tabela IV. Porcentagem consumida de vegetais nos diferentes dias de experimento, no decorrer do desenvolvimento de Podocnemis unifilis. (DP) Desvio padrão, (gl) graus de liberdade, (NS) não significativo.

\begin{tabular}{|c|c|c|c|c|c|c|}
\hline & $<1$ ano & I a 5 anos & $<1$ ano & 5 anos & 1 a 5 anos & $>5$ anos \\
\hline & 2,4 & 42,7 & 2,4 & 59,6 & 42,7 & 59,6 \\
\hline & 6,7 & 8,1 & 6,7 & 4,1 & 8,1 & 4,1 \\
\hline & 59,0 & 33,0 & 59,0 & 49,8 & 33,0 & 49,8 \\
\hline & 43,9 & 30,1 & 43,9 & 26,0 & 30,1 & 26,0 \\
\hline & 10,0 & 29,3 & 10,0 & 28,5 & 29,3 & 28,5 \\
\hline & 100,0 & 15,1 & 100,0 & 36,6 & 15,1 & 36,6 \\
\hline & 2,1 & 47,3 & 2,1 & 73,0 & 47,3 & 73,0 \\
\hline & 13,0 & 31,6 & 13,0 & 24,6 & 31,6 & 24,6 \\
\hline & 29,8 & 30,8 & 29,8 & 22,6 & 30,8 & 22,6 \\
\hline & 21,8 & 28,9 & 21,8 & 48,2 & 28,9 & 48,2 \\
\hline & 10,4 & 28,6 & 10,4 & 10,4 & 28,6 & 10,4 \\
\hline & 26,8 & 26,9 & 26,8 & 24,0 & 26,9 & 24,0 \\
\hline & 61,0 & 14,7 & 61,0 & 40,6 & 14,7 & 40,6 \\
\hline & 19,3 & 53,5 & 19,3 & 35,4 & 53,5 & 35,4 \\
\hline & 31,5 & 41,4 & 31,5 & 49,8 & 41,4 & 49,8 \\
\hline & 4,4 & 13,5 & 4,4 & 39,6 & 13,5 & 39,6 \\
\hline & 23,5 & 48,9 & 23,5 & 52,2 & 48,9 & 52,2 \\
\hline Observações & 17 & 17 & 17 & 17 & 17 & 17 \\
\hline Média & 27,4 & 30,8 & 27,4 & 36,8 & 30,8 & 36,8 \\
\hline DP & 26,02 & 13,04 & 26,02 & 17,71 & 13,04 & 17,71 \\
\hline gl & 16 & & 16 & & 16 & \\
\hline Estatística t & $-0,441$ & & $-1,241$ & & $-1,599$ & \\
\hline $\begin{array}{l}\mathrm{P}(\mathrm{T}<=\mathrm{t}) \\
\text { uni-caudal }\end{array}$ & $0,333 \mathrm{NS}$ & & $0,116 \mathrm{NS}$ & & $0,065 \mathrm{NS}$ & \\
\hline
\end{tabular}

do que $P$. unifilis nesta faixa etária. Obteve-se um aumento muito significativo ( $\mathrm{p}=0,005, \mathrm{SS}$ ) para a porcentagem de vegetais ingerida por $P$. expansa na dieta, em relação a $P$. unifilis (Tab. VI). Em média a dieta de $P$. expansa era constituída de $36,7 \%$ de alimentos de origem vegetal e a de $P$. unifilis, de $30,8 \%$.

O consumo de carne dos indivíduos do grupo G3 de $P$. unifilis e de $P$. expansa teve um aumento não significativo ( $\mathrm{p}=$ 0,086 , NS) porém o consumo de vegetais e também a quantidade total de alimentos ingeridos por P.expansa teve um aumento altamente significativo (respectivamente, $\mathrm{p}=$ 0,000005 e $\mathrm{p}=0,00002$, SSS), indicando que $P$. expansa come mais do que $P$. unifilis nesta faixa etária. A diferença na porcentagem de vegetais ingeridos por $P$. expansa em relação a P. unifilis (Tab. VII) foi altamente significativa ( $\mathrm{p}=0,000005$, SSS). A dieta de $P$. expansa consistiu de $51,6 \%$ de alimentos de origem vegetal contra $36,8 \%$ de $P$. unifilis. 
Tabela V. Teste t de Student comparando $P$. expansa e $P$. unifilis. (DP) Desvio adrão, (gl) graus de liberdade, (NS) não significativo.

\begin{tabular}{|c|c|c|c|c|c|c|}
\hline \multirow{3}{*}{$\begin{array}{c}\text { Ano } 1998 \\
10-\text { nov }\end{array}$} & \multicolumn{2}{|c|}{ Carne (g) } & \multicolumn{2}{|c|}{ Vegetais (g) } & \multicolumn{2}{|c|}{ Total $(\mathrm{g})$} \\
\hline & \multicolumn{2}{|c|}{ P. expansa P. unifilis } & \multicolumn{2}{|c|}{ P. expansa P. unifilis } & \multicolumn{2}{|c|}{ P. expansa P. unifilis } \\
\hline & 39,8 & 37,4 & 0,6 & 0,9 & 40,4 & 38,3 \\
\hline 11-nov & 49,8 & 62,3 & 15,6 & 4,5 & 65,4 & 66,7 \\
\hline 12-nov & 37,4 & 5,0 & 4,4 & 7,2 & 41,7 & 12,1 \\
\hline 13-nov & 24,9 & 7,5 & 5,5 & 5,8 & 30,4 & 13,3 \\
\hline 14-nov & 24,9 & 49,8 & 2,9 & 5,5 & 27,8 & 55,3 \\
\hline 22-nov & 7,5 & - & 6,5 & 8,1 & 13,9 & 8,1 \\
\hline 23-nov & 12,5 & 49,8 & 3,9 & 1,1 & 16,3 & 50,9 \\
\hline 25 -nov & 19,9 & 12,5 & 0,9 & 1,9 & 20,8 & 14,3 \\
\hline 27-nov & 37,4 & 24,9 & 11,9 & 10,6 & 49,3 & 35,5 \\
\hline 28-nov & 12,5 & 19,9 & 4,5 & 5,6 & 16,9 & 25,5 \\
\hline 29-nov & 37,4 & 49,8 & 3,1 & 5,8 & 40,4 & 55,6 \\
\hline 30-nov & 12,5 & 12,5 & 2,3 & 4,6 & 14,7 & 17,0 \\
\hline 05-dez & 24,9 & 24,9 & 17,8 & 39,0 & 42,7 & 63,9 \\
\hline 07-dez & 49,8 & 49,8 & 17,0 & 11,9 & 66,8 & 61,7 \\
\hline 08-dez & 49,8 & 49,8 & 15,4 & 22,9 & 65,2 & 72,7 \\
\hline 09-dez & 49,8 & 62,3 & 1,9 & 2,9 & 51,7 & 65,1 \\
\hline 11-dez & 74,7 & 62,3 & 16,3 & 19,1 & 91,0 & 81,3 \\
\hline Observações & 17 & 17 & 17 & 17 & 17 & \\
\hline Média & 33,2 & 34,1 & 7,7 & 9,2 & 40,9 & 43,4 \\
\hline DP & 18,13 & 21,86 & 6,37 & 9,72 & 22,13 & 24,36 \\
\hline gl & 16 & & 16 & & 16 & \\
\hline Estatística t & $-0,221$ & & $-1,011$ & & $-0,611$ & \\
\hline $\begin{array}{l}\mathrm{P}(\mathrm{T}<=\mathrm{t}) \\
\text { uni-caudal }\end{array}$ & 0,414 NS & & $0,16351 \mathrm{~N}$ & & $0,275 \mathrm{NS}$ & \\
\hline
\end{tabular}

O teste $t$ de Student revelou que, para os espécimes do grupo G2 de P. expansa, há um aumento significativo $(\mathrm{p}=0,013$, S) do consumo de carne, quando esta é colocada em área alagada. Com relação ao total de vegetais, não obtivemos um resultado estatisticamente significativo ( $p=0,223, \mathrm{NS})$. Na análise da quantidade total de alimentos (carne e vegetais) ocorre um aumento altamente significativo do consumo ( $\mathrm{p}=$ 0,0009 , SSS) quando a alimentação é colocada na água (Tab. VIII). Em média, foram consumidos $159,1 \mathrm{~g}$ de alimentos que foram colocados em área seca, contra 185,7 g que foram colocados na água.

Em P. unifilis o teste $t$ de Student para os espécimes do grupo G2 demonstrou um aumento significativo $(p=0,046, S)$ na quantidade de alimentos de origem vegetal ingerida, sendo esta maior quando eles são colocados em área seca. Porém, no
Tabela VI. Teste t de Student comparando o consumo de alimentos de origem animal e vegetal em $P$. expansa e em $P$. unifilis: (DP) Desvio padrão, (gl) graus de liberdade, (NS) não significativo, (S) significativo, (SS) muito significativo.

\begin{tabular}{|c|c|c|c|c|c|c|}
\hline \multirow{2}{*}{ Ano1999 } & \multicolumn{2}{|c|}{ Carne(g) } & \multicolumn{2}{|c|}{ Vegetais(g) } & \multicolumn{2}{|c|}{ Total(g) } \\
\hline & P.expansa & P.unifilis & P.expansa & P.unifilis & P.expansa & P.unifilis \\
\hline 9-nov & 124,5 & 124,5 & 119,2 & 92,6 & 243,7 & 217,1 \\
\hline 10-nov & 124,5 & 124,5 & 34,3 & 11,0 & 158,8 & 135,5 \\
\hline 11-nov & 124,5 & 124,5 & 79,4 & 61,4 & 203,9 & 185,9 \\
\hline 12-nov & 124,5 & 124,5 & 63,0 & 53,6 & 187,5 & 178,1 \\
\hline 13-nov & 124,5 & 124,5 & 63,7 & 51,7 & 188,2 & 176,2 \\
\hline 14-nov & 124,5 & 124,5 & 7,1 & 22,1 & 131,6 & 146,6 \\
\hline 22-nov & 44,8 & 74,7 & 91,0 & 67,0 & 135,9 & 141,7 \\
\hline 23-nov & 124,5 & 124,5 & 79,0 & 57,5 & 203,5 & 182,0 \\
\hline 25-nov & 124,5 & 124,5 & 63,6 & 55,5 & 188,1 & 180,0 \\
\hline 27-nov & 112,1 & 104,6 & 83,5 & 42,6 & 195,6 & 147,2 \\
\hline 28-nov & 124,5 & 124,5 & 41,2 & 49,9 & 165,7 & 174,4 \\
\hline 29-nov & 124,5 & 124,5 & 28,7 & 45,9 & 153,2 & 170,4 \\
\hline 30-nov & 124,5 & 124,5 & 28,6 & 21,5 & 153,1 & 146,0 \\
\hline 5-dez & 62,3 & 74,7 & 91,7 & 85,9 & 153,9 & 160,6 \\
\hline 7-dez & 124,5 & 124,5 & 117,1 & 87,9 & 241,6 & 212,4 \\
\hline 8-dez & 124,5 & 124,5 & 84,4 & 19,4 & 208,9 & 143,9 \\
\hline 11-dez & 124,5 & 124,5 & 119,2 & 119,2 & 243,7 & 243,7 \\
\hline Observações & 17 & 17 & 17 & 17 & 17 & 17 \\
\hline Média & 115,4 & 117,5 & 70,3 & 55,6 & 185,7 & 173,0 \\
\hline DP & 23,69 & 16,80 & 33,68 & 28,99 & 36,07 & 29,92 \\
\hline gl & 16 & & 16 & & 16 & \\
\hline Estatística t & $-1,053$ & & 2,970 & & 2,370 & \\
\hline $\begin{array}{l}\mathrm{P}(\mathrm{T}<=\mathrm{t}) \\
\text { uni-caudal }\end{array}$ & 0,154 NS & & 0,005 SS & & $0,015 \mathrm{~S}$ & \\
\hline
\end{tabular}

total geral de alimentação consumida, o resultado não é estatisticamente significativo ( $p=0,398, N S)$, apesar da média de alimentos ingeridos ser maior quando estes são colocados em área alagada (Tab. IX). Em média, foram consumidos 170,9g de alimentos que foram colocados em área seca contra 173,0 g que foram colocados na água.

Com relação a $P$. sextuberculata, esta espécie mostra-se muito mais carnívora do que $P$. expansa e $P$. unifilis. Como pode se observar nas tabelas X e XI, sua dieta é quase que exclusivamente de origem animal nos grupos G1 e G2. No grupo G1, $99 \%$ da dieta foi de alimentos de origem animal e $1 \%$ de origem vegetal e, no grupo $\mathrm{G} 2,99,5 \%$ e $0,5 \%$, respectivamente. 
Tabela VIII. Teste t de Student para preferência alimentar de indivíduos de Podocnemis expansa entre um e cinco anos em área alagada e seca. (DP) Desvio padrão, , (gl) graus de liberdade, (NS) não significativo, (S) significativo, (SSS) altamente significativo.

\begin{tabular}{|c|c|c|c|c|c|c|}
\hline \multirow{2}{*}{ Ano 1999} & \multicolumn{2}{|c|}{ Carne (g) } & \multicolumn{2}{|c|}{ Total vegetais $(\mathrm{g})$} & \multicolumn{2}{|c|}{ Total geral (g) } \\
\hline & Seca & Alagada & Seca & Alagada & Seca & Alagada \\
\hline 9-nov & 124,5 & 124,5 & 104,3 & 119,2 & 228,8 & 243,7 \\
\hline 10-nov & 99,6 & 124,5 & 24,2 & 34,3 & 123,8 & 158,8 \\
\hline 11-nov & 124,5 & 124,5 & 61,0 & 79,4 & 185,5 & 203,9 \\
\hline 12-nov & 124,5 & 124,5 & 54,6 & 63,0 & 179,1 & 187,5 \\
\hline 13-nov & 124,5 & 124,5 & 58,8 & 63,7 & 183,3 & 188,2 \\
\hline 14-nov & 124,5 & 124,5 & 42,1 & 7,1 & 166,6 & 131,6 \\
\hline 22-nov & 49,8 & 44,8 & 49,4 & 91,0 & 99,2 & 135,9 \\
\hline 23-nov & 79,7 & 124,5 & 48,7 & 79,0 & 128,3 & 203,5 \\
\hline 25-nov & 62,3 & 124,5 & 83,3 & 63,6 & 145,6 & 188,1 \\
\hline 27-nov & 124,5 & 112,1 & 84,8 & 83,5 & 209,3 & 195,6 \\
\hline 28-nov & 12,5 & 124,5 & 86,7 & 41,2 & 99,1 & 165,7 \\
\hline 29-nov & 49,8 & 124,5 & 43,1 & 28,7 & 92,9 & 153,2 \\
\hline 30-nov & 49,8 & 124,5 & 40,5 & 28,6 & 90,3 & 153,1 \\
\hline 5-dez & 62,3 & 62,3 & 55,8 & 91,7 & 118,1 & 153,9 \\
\hline 7-dez & 124,5 & 124,5 & 107,0 & 117,1 & 231,5 & 241,6 \\
\hline 8-dez & 124,5 & 124,5 & 67,6 & 84,4 & 192,1 & 208,9 \\
\hline 11-dez & 124,5 & 124,5 & 106,7 & 119,2 & 231,2 & 243,7 \\
\hline Observações & 17 & 17 & 17 & 17 & 17 & 17 \\
\hline Média & 93,3 & 115,4 & 65,8 & 70,3 & 159,1 & 185,7 \\
\hline DP & 38,02 & 23,69 & 25,33 & 33,68 & 50,62 & 36,07 \\
\hline gl & 16 & & 16 & & 16 & \\
\hline Estatística t & $-2,455$ & & $-0,783$ & & $-3,72$ & \\
\hline $\begin{array}{l}\mathrm{P}(\mathrm{T}<=\mathrm{t}) \\
\text { uni-caudal }\end{array}$ & $0,013 \mathrm{~S}$ & & $0,223 \mathrm{NS}$ & & 0,0009 SSS & \\
\hline
\end{tabular}

\section{DISCUSSÃO}

O comportamento alimentar de P.expansa, P.unifilis e P.sextuberculata é semelhante ao descrito por Molina (1991) para Phrynops geoffroanus (Schweigger, 1812). A dilaceração realizase sempre com o auxílio alternado dos membros dianteiros. Molina (1991) também aponta este aspecto para P. geoffroanus, salientando que, pela disposição anatômica, as espécies que pertencem à sub-ordem Pleurodira utilizam os membros anteriores de forma alternada. O autor também ressalta para algumas espécies da sub-ordem Criptodira, como Trachemys dorbignyi, que a dilaceração pode ocorrer com o auxílio dos membros anteriores, simultânea ou alternadamente.

Revista Brasileira de Zoologia 20 (1): 161-168, março 2003
Tabela IX. Teste t de Student para preferência alimentar de indivíduos de Podocnemis unifilis entre um e cinco anos em área alagada e seca. (DP) Desvio padrão, (gl) graus de liberdade, (NS) não significativo, (S) significativo.

\begin{tabular}{|c|c|c|c|c|c|c|}
\hline \multirow{2}{*}{ Ano1999 } & \multicolumn{2}{|c|}{ Carne } & \multicolumn{2}{|c|}{ Totalvegetais } & \multicolumn{2}{|c|}{ Totalgeral } \\
\hline & Seca & Alagada & Seca & Alagada & Seca & Alagada \\
\hline 9-nov & 124,5 & 124,5 & 84,2 & 92,6 & 208,7 & 217,1 \\
\hline 10-nov & 24,9 & 124,5 & 5,7 & 11,0 & 30,6 & 135,5 \\
\hline 11-nov & 124,5 & 124,5 & 62,0 & 61,4 & 186,5 & 185,9 \\
\hline 12-nov & 124,5 & 124,5 & 64,3 & 53,6 & 188,8 & 178,1 \\
\hline 13-nov & 124,5 & 124,5 & 45,4 & 51,7 & 169,9 & 176,2 \\
\hline 14-nov & 124,5 & 124,5 & 31,3 & 22,1 & 155,8 & 146,6 \\
\hline 22-nov & 87,2 & 74,7 & 75,1 & 67,0 & 162,2 & 141,7 \\
\hline 23-nov & 124,5 & 124,5 & 69,3 & 57,5 & 193,8 & 182,0 \\
\hline 25-nov & 124,5 & 124,5 & 69,6 & 55,5 & 194,1 & 180,0 \\
\hline 27-nov & 87,2 & 104,6 & 46,5 & 42,6 & 133,7 & 147,2 \\
\hline 28-nov & 124,5 & 124,5 & 28,0 & 49,9 & 152,5 & 174,4 \\
\hline 29-nov & 124,5 & 124,5 & 53,0 & 45,9 & 177,5 & 170,4 \\
\hline 30-nov & 62,3 & 124,5 & 52,1 & 21,5 & 114,4 & 146,0 \\
\hline 5-dez & 74,7 & 74,7 & 86,0 & 85,9 & 160,7 & 160,6 \\
\hline 7-dez & 124,5 & 124,5 & 105,7 & 87,9 & 230,2 & 212,4 \\
\hline 8-dez & 124,5 & 124,5 & 78,3 & 19,4 & 202,8 & 143,9 \\
\hline 11-dez & 124,5 & 124,5 & 119,2 & 119,2 & 243,7 & 243,7 \\
\hline Observações & 17 & 17 & 17 & 17 & 17 & 17 \\
\hline Média & 107,7 & 117,5 & 63,3 & 55,6 & 170,9 & 173,0 \\
\hline DP & 29,83 & 16,80 & 28,21 & 28,99 & 48,69 & 29,92 \\
\hline gl & 16 & & 16 & & 16 & \\
\hline Estatística t & $-1,441$ & & 1,798 & & $-0,263$ & \\
\hline $\begin{array}{l}\mathrm{P}(\mathrm{T}<=\mathrm{t}) \\
\text { uni-caudal }\end{array}$ & $0,084 \mathrm{NS}$ & & $0,046 \mathrm{~S}$ & & $0,398 \mathrm{NS}$ & \\
\hline
\end{tabular}

MoLina et al. (1998) descrevem as fases do comportamento alimentar em Phrynops hilarii (Duméril \& Bibron, 1835). O padrão geral é semelhante ao verificado nas espécies do presente estudo, no entanto, não observam o movimento dos membros anteriores sobre a cabeça indo em direção à boca como em $P$. expansa, $P$. unifilis e $P$. sextuberculata, relacionados provavelmente como a retirada de restos alimentares da boca.

Molina (1991) e Molina et al. (1998) apontam em $P$. geffroanus e em $P$. hilarii o cleptoparasitismo, que consiste na captura de alimentos já apreendidos por outros indivíduos. Este comportamento também foi observado nas espécies aqui estudadas. 
Tabela X. Podocnemis sextuberculata. Preferência alimentar de 14 indivíduos com menos de um ano, na Fundação Parque Zoológico de São Paulo no período de 16.IV.1997 a 17.VII.1997.

\begin{tabular}{lcccc}
\hline \multirow{2}{*}{ Descrição } & \multicolumn{2}{c}{ Alimento oferecido } & & \multicolumn{2}{c}{ Alimento consumido } \\
\cline { 5 - 5 } \cline { 5 - 5 } Origem animal & Unidades & & Unidades & $\%$ \\
\hline Carne & & & & \\
Sardinha & 150 & & 43,1 & 28,73 \\
\hline Total & 140 & & 52,5 & 37,5 \\
\hline Origem vegetal & 290 & 95,6 & 32,97 \\
Tomate & 140 & & 0,2 & 0 \\
Mamão & 150 & & 0,2 & 0 \\
Banana & 150 & & 0,4 & 0 \\
Almeirão & 140 & & - & - \\
Cenoura & 130 & & - & - \\
Abóbora & 90 & & - & - \\
Repolho & 10 & & - & - \\
Laranja & 40 & 0,2 & 0,01 \\
\hline Total & 850 & 1 & 0,12 \\
\hline
\end{tabular}

Tabela XI. Preferência alimentar de indivíduos entre um e cinco anos de Podocnemis sextuberculata.

\begin{tabular}{|c|c|c|c|}
\hline \multirow{2}{*}{ Descrição } & \multirow{2}{*}{$\frac{\text { Alimento oferecido }}{\text { Unidades }}$} & \multicolumn{2}{|c|}{ Alimento consumido } \\
\hline & & Unidades & $\%$ \\
\hline \multicolumn{4}{|l|}{ Origem animal } \\
\hline Carne & 160 & 36,4 & 22,75 \\
\hline \multicolumn{4}{|l|}{ Origem vegetal } \\
\hline Tomate & 130 & - & - \\
\hline Couve & 50 & - & - \\
\hline Banana & 60 & - & - \\
\hline Cenoura & 90 & - & - \\
\hline Acelga & 30 & - & - \\
\hline Maçã & 110 & - & - \\
\hline Repolho & 80 & 0,1 & 0,13 \\
\hline Laranja & 40 & 0,1 & 0,25 \\
\hline Total vegetal & 590 & 0,2 & 0,03 \\
\hline
\end{tabular}

BeLKIN \& Gans (1968) descreveram o comportamento de neustofaia em $P$. unifilis. Este mesmo comportamento foi observado no presente estudo para os grupos G2 e G3 de $P$. unifilis. No entanto, ão foi verificado este mecanismo alimentar no grupo G1 e também nos representantes de $P$. expansa e $P$. sextuberculata. Molina (1991) e Molina et al. (1998) não constataram esta estratégia alimentar para $P$. geoffroanus e para $P$. hilarii. Monge-NÁjera \& BRENEs (1987) ressaltam a visão como o principal sentido utilizado na localização e no reconhecimento do alimento em Kinosternon scorpioides (Linnaeus, 1766). Nas espécies aqui estudadas, verificou-se que o olfato, provavelmente, também tem papel fundamental nestas etapas, pois ao se oferecer carne ou sardinha fora do campo visual do animal, este apresentava o comportamento de aproximação. Caso a alimentação fosse constituída apenas de frutas e verduras, os exemplares não apresentariam este comportamento. Mora \& Rugeles (1981) relatam que, em cativeiro, Geochelone carbonaria (Spix, 1824) e G. denticulata (Linnaeus, 1766) utilizam o olfato e a visão para localizar o alimento.

O movimento denominado de estocada, ou seja, um rápido movimento de pescoço e cabeça para realizar a apreensão do alimento, é ressaltado por PRITCHARD (1984) para alguns representantes das famílias Emydidae, Chelydridae, Trionychidae e Chelidae. Neste estudo, a apreensão ocorre por abocanhamento do alimento, sem esse rápido movimento de estocada descrito na literatura. LegLer (1993) relatam que, em Chelodina expansa (Gray, 1857) e em Chelus, as fases de apreensão e ingestão da presa são muito rápidas para a visão humana acompanhar. Em C. expansa, presas com tamanho adequado podem passar pela boca sem tocar as maxilas e, muitas vezes, chegam vivas o estômago.

Com relação à preferência alimentar, verifica-se que $P$. sextuberculata demonstra ser uma espécie predominantemente carnívora nas faixas etárias estudadas, que incluem representantes jovens e recém eclodidos. Já $P$. expansa e $P$. unifilis podem ser consideradas espécies onívoras, pois em todas as fases de desenvolvimento aceitaram alimentos de origem animal e vegetal. Legler (1993) comenta que a maioria das espécies de Testudines é onívora oportunista.

No presente trabalho, verifica-se uma variação da dieta durante o desenvolvimento de $P$. expansa, ocorrendo um aumento significativo na porcentagem de alimentos de origem vegetal, à medida que aumenta a faixa etária do animal. Comparando os grupos G1 e G2, a média da porcentagem de vegetais na dieta sobe de $19,1 \%$ para $36,7 \%$, respectivamente, sendo este aumento muito significativo $(\mathrm{p}<0,01)$. Comparando os grupos G2 e G3, a média da porcentagem de alimentos de origem vegetal na dieta sobe de $36,7 \%$ para $51,6 \%$, sendo este aumento altamente significativo ( $\mathrm{p}<0,001)$ (Tab. III). PrITCHARD $\&$ Trebbau (1984) ressaltam que jovens de P. expansa em cativeiro preferem alimentos de origem animal.

Em $P$. unifilis o aumento do consumo de alimentos de origem vegetal ao longo do desenvolvimento não é estatisticamente significativo. A porcentagem média do consumo de vegetais na dieta é de 27,4\% em G1, 30,8\% em G2 e $36,8 \%$ em G3 (Tab. IV). Portanto, em cativeiro P. expansa apresentou-se mais herbívora do que $P$. unifilis considerando o decorrer de seu desenvolvimento. Quando comparam-se os grupos G1 de $P$. expansa e $P$. unifilis, não há variação na quantidade de alimentos de origem vegetal e animal ingerida por estas espécies (Tab. V). Já comparando os grupos G2 destas espécies, a porcentagem de vegetais ingerida tem um aumento muito significativo ( $\mathrm{p}<0,01)$, sendo maior em P. expansa (Tab. VI). Comparando os grupos G3 entre as duas espécies, a porcentagem de consumo vegetal tem um aumento muito significativo $(\mathrm{p}<0,001)$ em $P$. expansa. 
Os exemplares de $P$. sextuberculata mantiveram-se, nos grupos G1 e G2, predominantemente carnívoros. No grupo G1, $1 \%$ de sua dieta foi de origem vegetal e no grupo G2, $0,5 \%$ (Tabs X e XI). ERnst \& Barbour (1989) indicam que, na natureza, esta espécie se alimenta de plantas aquáticas e peixes.

A quantidade total de alimentos que foi consumida por P. expansa foi maior e altamente significativa $(\mathrm{p}<0,001)$ quando a alimentação foi colocada na água. O total ingerido de vegetais não variou, porém, houve uma diminuição do consumo de carne que foi colocada em área seca, ou seja, fora da água (Tab. VIII). Isso indica que, para os criatórios com finalidade comercial onde, em geral, a alimentação é colocada fora da água para facilitar o manejo do cativeiro, é possível que a quantidade de alimentação ingerida esteja sendo afetada. $\mathrm{O}$ mesmo não ocorre em $P$. unifilis, pois o consumo geral dos alimentos não tem uma diminuição significativa, mesmo quando colocados fora da água. Foi verificado inclusive um aumento significativo $(\mathrm{p}<0,05)$ na quantidade ingerida de vegetais colocados em área seca quando comparada com os colocados em área alagada (Tab. IX). Isso indica que não há problema em colocar a alimentação fora da água para P. unifilis, sendo esta espécie menos sensível a esta alteração.

\section{CONCLUSÕES}

As principais fases do comportamento alimentar de Podocnemis expansa, $P$. unifilis e $P$. sextuberculata, quando a alimentação é constituída por presas vivas, são: forrageio, perseguição, apreensão, dilaceração e ingestão.

As fases do comportamento alimentar de $P$. expansa, $P$. unifilis e $P$. sextuberculata, quando a alimentação é constituída por alimento sem mobilidade, são: forrageio, aproximação, reconhecimento olfativo, apreensão, dilaceração e ingestão.

Cleptoparasitismo ocorre nas três espécies e o mecanismo de neustofagia foi verificado apenas em $P$. unifilis.

Podocnemis sextuberculata mostrou-se uma espécie predominantemente carnívora em cativeiro, enquanto $P$. expansa e $P$. unifilis mostraram-se onívoras.

Podocnemis expansa pode ser considerada mais herbívora do que $P$. unifilis em cativeiro nas faixas etárias de um a cinco anos e maior de cinco anos.

Podocnemis expansa é uma espécie mais sensível do que $P$. unifilis com relação à alteração do local em que se coloca o alimento, diminuindo o seu consumo caso este seja colocado fora da água.

\section{AGRADECIMENTOS}

Ao Instituto de Biociências da Universidade de São Paulo, em especial à pessoa da Profa. Dra. Erika Schlenz, pelo apoio e incentivo. À Fundação Parque Zoológico de São Paulo, em especial ao Sr. Mário Borges da Rocha, e ao Centro Nacional dos Quelônios da Amazônia, CENAQUA/IBAMA, pelo apoio e material fornecidos. À Representação do IBAMA no Estado do Tocantins pela disponibilização da Base Física do Parque Nacional do Araguaia. À Fundação Universidade do Tocantins (UNITINS), especialmente ao Campus Universitário de Araguaína, por todo o apoio recebido durante este trabalho. Aos alunos da UNITINS, Giovanni Salera Junior e Eliene Santos Reis. À CAPES, pela concessão da bolsa durante a primeira fase do trabalho.

Recebido em 20.V.2002; aceito em 17.III.2003.

\section{REFERÊNCIAS BIBLIOGRÁFICAS}

Altmann, J. 1974. Observational Study of Behaviour: Sampling Methods. Behaviour, Leiden, 49 (3-4): 228-267.

Belkin, D.A. \& C. Gans. 1968. An Usual Chelonian Feeding Niche. Ecology, New York, 49 (4): 768-769.

Carpenter, C.C. \& G.W. Ferguson. 1977. Variation and Evolution of Stereotyped Behavior in Reptiles, p. 335-554. In: C. Gans $\&$ D.W. TinKLE (Eds). Biology of the Reptilia. Michigan, Academic Press, 720p.

Clark, D.B. \& J.W. Gibbons. 1969. Dietary Shift in the Turtle Pseudemys scripta (Schoepff) from Youth to Maturity. Copeia, New York, (4): 740-705.

Ernst, C.H. \& R.W. Barbour. 1989. Turtles of the World. Washington, D.C., Smithsonian Institution Press, 313p.

GoIN, C.J. \& O.B. GoIN. 1962. Introduction to Herpetology. San Francisco, W.H. Freeman, 341p.

HaRT, D.R. 1983. Dietary and Habitat Shift with Size of Redeared Turtles (Pseudemys scripta) in a Southern Louisiana Population. Herpetologica, Chicago, 39 (3): 285-290.

Legler, J.M. 1993. Morphology and Physiology of the Chelonia, p. 108-119. In: C.J. Glasby; G.J.B. Ross \& P.L. Beesley (Eds). Fauna of Austrália. Canberra, vol. 2, 439p.

Molina, F.B. 1991. Observações sobre os Hábitos e o Comportamento Alimentar de Phrynops geoffroanus (Schweigger, 1812) em Cativeiro (Reptilia, Testudines, Chelidae). Revista Brasileira de Zoologia, Curitiba, 7 (3): 319-326.

Molina, F.B.; M.B. Rocha \& L.A.B.M. Lula. 1998. Comportamento Alimentar e Dieta de Phrynops hilarii (Duméril \& Bibron) em Cativeiro (Reptilia, Testudines, Chelidae). Revista Brasileira de Zoologia, Curitiba, 15 (1): 73-79.

Monge-Nájera, J. \& B.M. Brenes. 1987. Notes on the Feeding Behavior of a Juvenile Mud Turtle Kinosternon scorpioides. Herpetological Review, Cincinati, 18 (1): 7-8.

Mora. O.V.C. \& M.L. Rugeles. 1981. Estudio Comparativo del comportamiento de dos especies de Morrocoy: Geochelone carbonaria y Geochelone denticulata y aspectos comparables de su morfologia externa. Cespedesia, Cali, 10 (37-38): 55122.

MoreirA, G.R.S. \& J.A.S. Loureiro. 1992. Contribución al Estudio de la Morfologia del Tracto Digestivo de Indivíduos Jóvenes de Podocnemis expansa (Testudinata: Pelomedusidae). Acta Zoologica Lilloana, Tucumán, 41: 345-348.

Pritchard, P.C.H. 1984. Piscivory in Turtles, and Evolution of the Long-necked Chelidae. In: M.W.J. Fergunson (ed.). Symposia of the Zoological Society of London, London, (52): 87-110.

Pritchard, P.C.H. \& P. Trebbau. 1984. The Turtles of Venezuela. Athens, Society for the Study of Amphibians and Reptiles, $403 p$.

SoKal, R.R. \& F.J. Rohlf. 1981. Biometry- The Principle and Practice of Statistics in Biological Research. San Francisco, W.H. Freeman, $2^{\text {nd }}$ ed., 859 p.

Teran, A.F.; R.C. Vogt \& M.F.S. Gomez. 1995. Food Habits of an Assemblage of Five Species of Turtles in the Rio Guaporé, Rondônia, Brazil. Journal of Herpetology, Columbus, 29 (4): 536-547. 